\title{
A Flexible and Low-Cost Tactile Sensor for Robotic Applications
}

\author{
Felix Sygulla, Felix Ellensohn, Arne-Christoph Hildebrandt, Daniel Wahrmann and Daniel Rixen
}

\begin{abstract}
For humans, the sense of touch is essential for interactions with the environment. With robots slowly starting to emerge as a human-centric technology, tactile information becomes increasingly important. Tactile sensors enable robots to gain information about contacts with the environment, which is required for safe interaction with humans or tactile exploration. Many sensor designs for the application on robots have been presented in literature so far. However, most of them are complex in their design and require high-tech tools for their manufacturing. In this paper, we present a novel design for a tactile sensor that can be built with low-cost, widely available materials, and low effort. The sensor is flexible, may be cut to arbitrary shapes and may have a customized spatial resolution. Both pressure distribution and absolute pressure on the sensor are detected. An experimental evaluation of our design shows low detection thresholds as well as high sensor accuracy. We seek to accelerate research on tactile feedback methods with this easy to replicate design. We consider our design a starting point for the integration of multiple sensor units to a large-scale tactile skin for robots.
\end{abstract}

\section{INTRODUCTION}

While today's industrial robots present potential harm and are therefore restricted by safety fences, the next generation of robots will have to provide intrinsic safety without external measures. This enables operation in human environments and an expansion of the field of robotic applications is expected, e.g. to health-care and service robotics or human-robot cooperation. In these scenarios, the robots must be able to handle unstructured, unknown and/or changing environments, which requires to make contact with the environment or at least to detect contacts, if they occur. There are many approaches to solve the safety and motion planning problems in such environments. Typically, these rely on visual information, prior-knowledge of objects, or single force/torque sensors, e.g. in the joints of a robot. However, these methods generally do not perform well in case the line of sight is obscured or unknown objects (with unknown mechanical properties) are encountered. With pure use of traditional force/torque sensors there is only limited information on the pressure distribution (contact positions), and contacts are generally avoided. The sense of touch is crucial for the exploration of unknown and unstructured environments, as contacts with the environment can be accurately detected and localized. In this paper, we propose three different designs for a tactile sensor, which are analyzed and compared. A final prototype is then selected based on experimental results, see Fig. 1. The design goals and prototypes are described in Sec. III An experimental evaluation is done in Sec. IV] A discussion on the results in Sec. $\mathrm{V}$ concludes our paper.

Chair of Applied Mechanics, Technical University of Munich, Boltzmannstr. 15, 85748 Garching, Germany felix. sygulla@tum. de

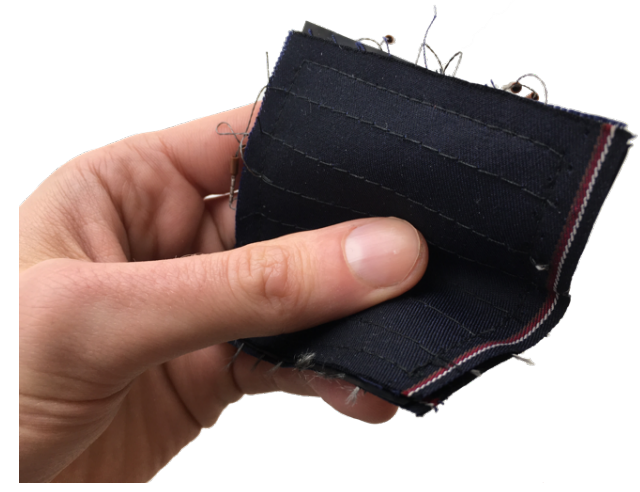

Fig. 1: Picture of the proposed tactile sensor (prototype $C$ )

\section{RELATED WORK}

Tactile sensing has been of increasing scientific interest in the last years. Numerous tactile sensor skins have been proposed [1]-[3]. Work has been done in the field of multimodal and modular tactile skins [4], [5] and on the problems of electric wiring and scalability [6], [7]. However, these tactile sensors are stiff and their application to arbitrarily shaped geometries is limited. Therefore, several 3D shaped sensor elements have been designed [8], [9]. While these sensors can be manufactured to fit arbitrary shapes, they are not inherently flexible. A flexible sensor simplifies integration and allows adaptation to any form of external contact. A completely flexible multi-modal skin based on a capacitive measurement principle is proposed in [10]. The design yields high accuracy and low cost. However, spatial resolution may be limited. In [11]-[13] sensor points - also called taxels are also based on larger and/or customized areas. While these sensors provide excellent results for specific applications, the theoretically reachable spatial resolution is limited.

To reach higher spatial resolutions, micro-mechanical taxels or a matrix structure of the connecting wires are used [14]-[19]. Most of these designs are based on flexible PCBs or injection-molded elastomers. Either the structure is complex or the manufacturing process targets high volumes. For low volumes and custom pieces, as needed in e.g. research labs, manufacturing effort and costs are usually high.

There are flexible sensor designs already described in literature, which can in general be produced easily. In [20] a robust and low-cost tactile skin is proposed, based on a capacitive measurement principle. In general, this design can be built easily without need for special machinery. However, it requires a dedicated circuit for each taxel, which complicates the overall design. Although the sensor could 
be made flexible, to the authors' knowledge no flexible version has been published so far. [21] presents an innovative approach based on a screen-printed piezoresistive ink and a flexible PCB. It is unclear though, how accurate the pressure measurements are and how easy the manufacturing process is. Another approach is based on electrical wires stitched into pressure conductive rubber [22]. The design is easy to build and flexible. Still, the accuracy of the sensor patch is unknown. In [23] a promising design based on a tactile matrix is proposed. It consists of carbon-blackfilled silicone and conductive fabric. Manufacturing is easy given the mix of carbon-black and silicone is known. The accuracy of the sensor is high, however it is unclear if higher spatial resolutions can be reached easily. Another approach is presented in [24], based on conductive ink on a PET film. While this sensor is easy to manufacture, only discrete pressure values are detected. In [25] a highly scalable tactile skin is presented, based on photo-reflectors covered by an urethane foam. The design is low-cost and relatively easy to build, but spatial resolution is limited by the size of the photo-reflectors.

All presented sensor designs suffer from drawbacks of some kind, namely rigidity, high costs, low spatial resolution or a complex manufacturing process. In this work, we focus on solving all of theses issues in one sensor design.

\section{Design OF THE TACTILE SENSOR}

In the following, the underlying design criteria, materials, and design variants of our sensor are described.

\section{A. Design Goals}

To determine the requirements for the tactile sensor, we consider several use cases, which are described shortly in the following. (1) Tactile sensing on robotic fingers with the ability to have a high spatial resolution $(2-3 \mathrm{~mm})$. (2) A flexible tactile skin, which may cover the whole body of a humanoid robot to detect collisions with the environment as well as the precise contact location. (3) A tactile foot sole, which is placed on the feet of a humanoid robot to detect the contact state. Based on these applications, the design goals for the tactile sensor are defined.

In our opinion, essential requirements are low cost and the ability to manufacture the sensor element with commonly available tools and materials. This allows to get more robots equipped with tactile sensing capabilities and therefore boost the development of tactile sensing strategies. For good integration on arbitrarily shaped surfaces, the material must be flexible and it must be possible to customize the shape and spatial resolution of the sensor.

The main objective of the sensor is to provide accurate positions for contacts on the sensor surface. Interpolation algorithms may be used to improve the overall resolution for a given amount of taxels. These algorithms require the pressure information and work best if linearity is given. Therefore, we think that the pressure accuracy of these sensors is important to achieve higher spatial resolutions. The calibration of the sensor should be easy and ideally only be carried out once.

\section{B. General Concept and Materials}

Conformance with the requirements is largely influenced by the selection of the used materials. In the following, the general concept and materials are motivated and described. To enable support for static as well as dynamic pressure measurements, the sensor is based on the piezoresistive effect. If pressure is applied to such a piezoresistive material, its electrical resistance decreases. The resistance is measured via conductive electrodes on both sides of the sensor-layer. To achieve a high spatial resolution, many such taxels are arranged next to each other. In order to keep the number of necessary connecting wires low, we use the commonlyknown matrix-structure. This means all electrodes on a vertical line are connected to one wire, and all electrodes on a horizontal line are connected to another common wire. By selecting the right combination of vertical and horizontal wires, the resistance for each taxel in the matrix can be measured.

Based on the design goals, we restricted ourselves to the use of commonly available and low-priced materials. We used retail prices to evaluate the overall costs. For larger volumes, costs may be significantly lower. The search led to the Velostat ${ }^{\mathrm{TM}} 4540$ EVA Copolymer $\left(\approx 50 € / \mathrm{m}^{2}\right)$, which is produced by $3 \mathrm{M}$ and consists of a polymeric foil (11 $\mu \mathrm{m}$ thickness) impregnated with carbon black. While this material is intended for packaging of electrostatic-sensitive devices, it is known for its piezoresistivity. For the electrodes, which also serve as connecting wires, we use a stainless steel thread (2-ply and 3-ply), which is also commonly available and costs $\approx 0,26 € / \mathrm{m}$. An outer protection layer is made of a cotton fabric $\left(\approx 3 € / \mathrm{m}^{2}\right)$, or neopren $\AA^{1}\left(\approx 25 € / \mathrm{m}^{2}\right)$.

\section{Sensor Prototypes}

We built and evaluated several prototypes for the overall assembly of the sensor element. In the following, the three best designs in terms of accuracy, manufacturability and low force thresholds, are described. All other tested prototypes and their drawbacks are described shortly in the Appendix

1) Woven Matrix with Glued Neoprene Cover $(A)$ : The structural composition of this prototype is depicted in Fig. 2 for a 3x3 taxels matrix. Stainless steel threads (3-ply) are woven into the polymeric foil to build a grid. This technique has been previously used in [22] for a silicone-rubber based matrix. Each vertical and horizontal thread intersects at exactly one point, where one of the threads is on the upper side of the polymer and the other on the lower side. On the left side of Fig. 2, one taxel on the matrix is magnified. The sewing process is carried out manually. The sensor element is wrapped in a neoprene cover to protect it from mechanical damage. The cover is glued to the inner layers using a silicone-based adhesive ${ }^{2}$ to preserve its flexibility. Before the cover is glued, a thin and flexible adhesive insulating foil is

\footnotetext{
${ }^{1}$ SEDO Chemicals Neoprene LS

${ }^{2}$ Dow Corning 3140 MIL-A-46146 RTV
} 


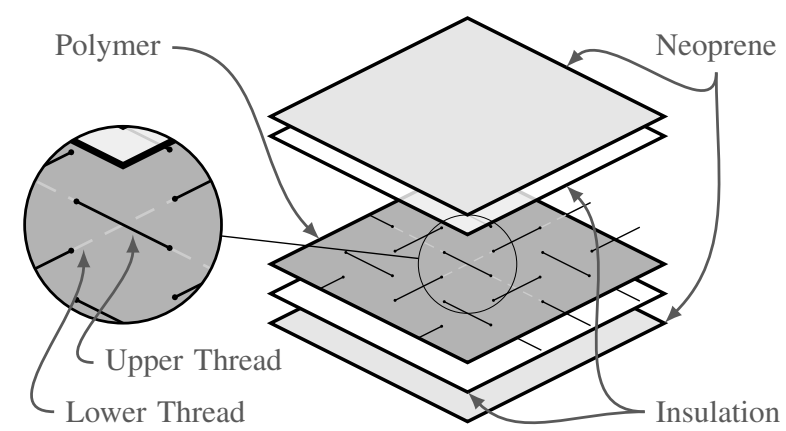

Fig. 2: Assembly of the sensor prototypes $A$ and $B$ with neoprene cover. For prototype $B$, the adhesive insulation layer is not present and all layers are sewed together.

attached to the polymer to protect the electrical contacts from the silicone adhesive, which may break the electrical contact between polymeric foil and stainless steel thread. This design allows a bending radius $r_{A} \approx 10 \mathrm{~mm}$.

2) Woven Matrix with Hand-Sewed Neoprene Cover (B): This prototype is similar to prototype $A$. Instead of gluing the neoprene cover, all layers are sewed together manually on the outer rim of the sensor patch. This way there are no adhesive tangential forces between the single layers even if the sensor is bended or deformed in any way. Without adhesive, the insulation layer is not necessary and a lower bending radius $r_{B} \approx 6 \mathrm{~mm}$ is reached.

3) Machine-Sewed Matrix with Fabrics Cover (C): The assembly of prototype $C$ is depicted in Fig. 3 for a $4 \times 4$ taxels matrix. In this case, the conductive threads are machinesewed into the protective cotton fabric. By using a lockstitch with a normal sewing thread and a stainless steel thread (2ply), the outer sides of the prototype stay electrically insulated from the contacts whereas the inner sides make contact to the polymer. All layers are machine-sewed together at the outer rim of the sensor patch. The result is a very flexible sensor prototype. Due to the machine-based sewing process, higher spatial resolutions and accuracies can be reached compared to prototypes $A$ and $B$. Furthermore, this prototype can be cut to fit arbitrary shapes after manufacturing, as long as the conductive threads can be connected. The position of the taxels can be designed arbitrarily (curves or other shapes) and/or non-uniformly throughout the patch. The sewing process is easy and fast. To provide compliance, an additional neoprene layer may be sewed to the bottom of the sensor patch. This prototype allows a bending radius of $r_{C} \approx 5 \mathrm{~mm}$.

\section{Calibration Model}

To reconstruct a pressure value from the resistance of each taxel, a calibration model must be identified. Due to the nature of the piezoresistive effect, the relation between resistance and pressure is highly nonlinear. We considered the following equations as possible models for the calibration of the sensor

$$
p=a \cdot e^{b \cdot R}
$$

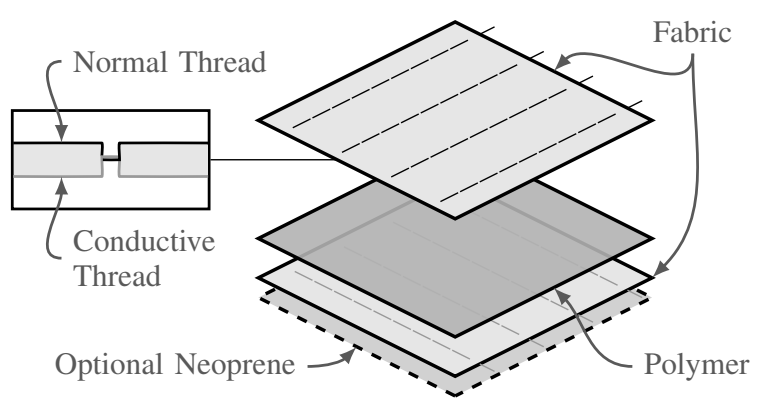

Fig. 3: Assembly of the sensor prototype $C$ with MachineSewed Matrix and Fabrics Cover

$$
\begin{aligned}
& p=a \cdot e^{b \cdot R}+c \cdot e^{d \cdot R} \\
& p=a \cdot R^{2}+b \cdot R+c,
\end{aligned}
$$

with the sensed pressure $p$, the resistance $R$ and the parameters $a, b, c, d$. Experimental results showed lowest fitting errors for the model described by Eq. (2). We use this model in two different ways to describe the sensor behavior.

1) Specific Model: The specific model uses a separate set of parameters for each taxel. This allows highest accuracies, but requires taxel-wise calibration.

2) Universal Model: The universal model uses only one set of parameters for all taxels. As all taxels have slightly different properties, higher errors occur. We seek to minimize calibration effort as the calibration data of few taxels may be sufficient to get a good model. Furthermore, a single set of parameters may be used for different units of the same sensor type.

\section{RESUlTS}

To evaluate the performance of the three different concepts, prototypes were built with a matrix-size of $6 \times 6$ taxels and $7 \mathrm{~mm}$ taxel distance. Each of these prototypes is analyzed in terms of measuring accuracy and response. Prototype $C$ is used with the additional neoprene layer at the bottom.

\section{A. Test Setup}

We tested the sensor prototypes using our manual force test stand IMADA HV-500N II combined with a Sauter FL20 force gauge. The accuracy of this reference force sensor is $\pm 0.05 \mathrm{~N}$, with a resolution of $0.01 \mathrm{~N}$. Furthermore we use a flat circular test probe with a contact area of $A_{p}=3.0 \mathrm{~cm}^{2}$. To read out the resistance of the sensor patch taxels, a custom electronics board with a voltage divider and several multiplexers is used. Data acquisition is done with a dSpace DS1103 controller board, which runs a Simulink model to scan the entire sensor matrix taxel per taxel and store the resistance information. We currently use a sample time of $0.001 \mathrm{~s}$ per taxel, which yields an update rate of $\approx 28 \mathrm{~Hz}$ for the entire matrix.

\section{B. Evaluation of the Sensor Patches}

During the evaluation process of the prototypes, a single taxel is loaded with a continuously increasing pressure until $33.33 \mathrm{kPa}(10 \mathrm{~N})$ are reached. Then, the force is decreased to 


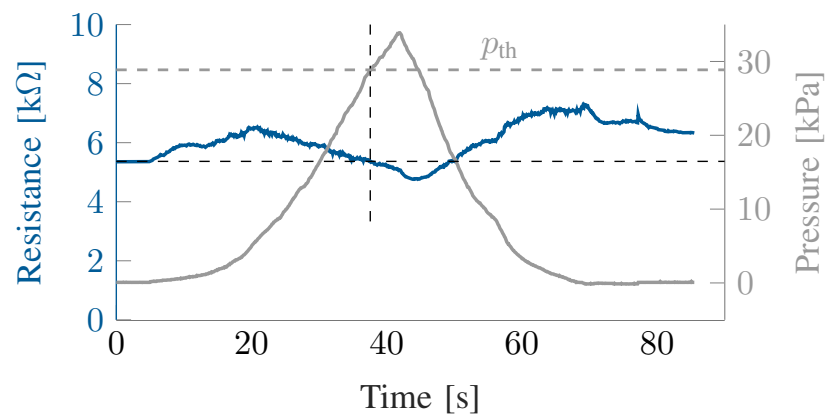

Fig. 4: Resistance and reference pressure over time for a taxel of prototype $A$

\begin{tabular}{lllll}
\hline & $A$ & $B$ & $C$ & \\
\hline $\max \left(p_{\text {th }}\right)$ & 27.9 & 3.9 & 0.7 & {$[\mathrm{kPa}]$} \\
$e_{\text {med,spec }}$ & 0.5 & 0.5 & 0.3 & {$[\mathrm{kPa}]$} \\
$\sigma_{15, \text { spec }}$ & 552 & 344 & 286 & {$[\Omega]$} \\
$e_{\text {med,univ }}$ & 4.1 & 2.7 & 1.5 & {$[\mathrm{kPa}]$} \\
\hline
\end{tabular}

TABLE I: Pressure thresholds and model errors for all prototypes

zero. The resistance of the corresponding taxel is measured during the whole process, which is repeated for every taxel on the matrix. Fig. 4 shows the result for one taxel of prototype $A$. For low loads the resistance of the taxel increases unexpectedly. This effect has been previously described in [26] and is caused by different elasticity of the stainless steel thread and the polymeric foil with its viscoelastic neoprene cover. Under load, the viscoelastic parts (polymeric foil and neoprene) move slowly. As the elasticity of the steel thread is higher, it lifts off the polymeric foil and the contact resistance increases. As a consequence, pressures below a specific pressure threshold $\left(p_{\text {th }}\right)$ cannot be detected reliably. The pressure value at the first point in time, where the resistance drops below the unloaded resistance is defined as $p_{\text {th }}$.

Prototype $B$ was originally designed to allow better sensor values in case the whole patch was bent or placed on a curved surface. However, without adhesive, the effect of increasing resistance is also clearly reduced and lower pressures can be detected. As horizontal movement between the layers is no longer constrained, the viscoelastic materials may deform horizontally, which possibly reduces the vertical deformation and therefore the lift-off of the steel threads.

The layers of Prototype $C$ are also not glued together. Furthermore, the cover is not made out of the viscoelastic neoprene, but a cotton fabric. As a consequence, even lower minimum pressures are reached. Therefore, prototype $C$ is selected as the final prototype and is considered in the following experiments.

\section{Calibration and Pressure Resolution}

In order to reconstruct pressure signals from the resistance on each taxel, calibration curves are identified from the measurements described in Sec. IV-B For this we take

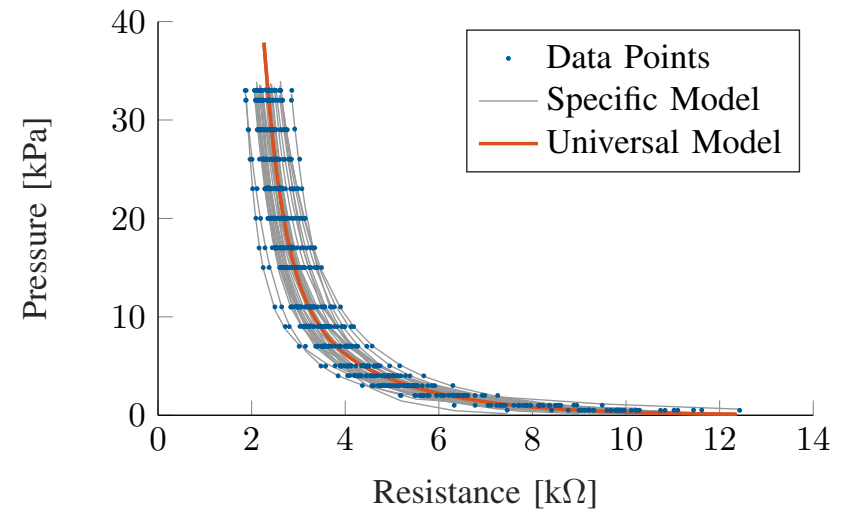

Fig. 5: Data points as well as specific models and universal model of prototype $C$

17 data points (reference pressure and resistance) between $0.5 \mathrm{kPa}$ and $33 \mathrm{kPa}$ of the data measured during one pressure increase. Note that we do not use data points with a resistance value above the unloaded resistance. Curve fitting to the calibration model in Sec. III-D is done using a nonlinear least-squares algorithm. To generate the universal model with a single set of parameters from the taxel-specific models, we use the median of each parameter. This results in a fit, which may have higher errors for outliers than a new least-squares fit over all data points, but has lower errors for most taxels (typical taxels). Results on the calibration of the prototypes as well as the maximum pressure thresholds $\max \left(p_{\text {th }}\right)$ of all taxels are shown in Tab. II For each taxel, the absolute error between the specific model and the data points is computed. The median of all these errors $e_{\text {med,spec }}$ is quite low for all three prototypes. The standard deviation of the resistance value at $15 \mathrm{kPa}$ for all specific parameter sets $\sigma_{15 \text {,spec }}$ is used to identify differences in the response of all taxels on a prototype. This means the specific calibration curves of prototype $A$ differ more from each other than those of prototype $B$. The lowest value for $\sigma_{15 \text {,spec }}$ is reached with prototype $C$. This also reflects in the median model error for a universal parameter set, $e_{\text {med,univ }}$, where prototype $C$ is best. The data points for every taxel, the specific models as well as the universal model over all taxels for prototype $C$ are shown in Fig. 5 Due to the nonlinear response of the material, the error in the universal model is best at low pressures with increasing uncertainty for higher pressures. In Fig. 6 the sensed pressure signal with specific- and universal parameter set is shown for prototype $C$ on a typical taxel, i.e. a taxel with a parameter set near the median of all taxels. Note that this is based on a measurement which is not part of the calibration data and contains different loading speeds as well as loads $(0-74 \mathrm{kPa})$ significantly above the calibration range $(0-33 \mathrm{kPa})$. Especially for low pressures the sensor values represent meaningful information about the intensity of a contact with the environment. In addition, there is no significant dependency on the loading speed, and the calibration model also yields reasonably accurate estimates outside the calibration range. In Tab. II the maximum sensor 


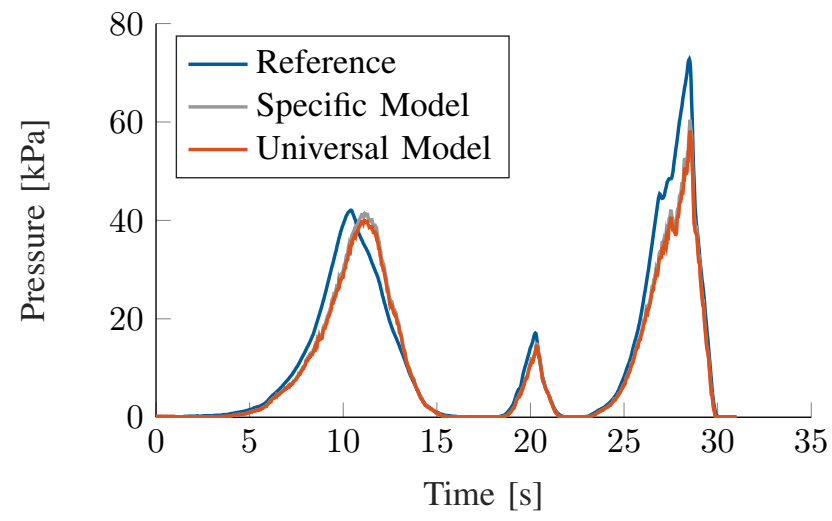

Fig. 6: Comparison of reference and sensed pressure for specific and universal model of prototype $C$

\begin{tabular}{lllll}
\hline Range: & $0-5 \mathrm{kPa}$ & $5-10 \mathrm{kPa}$ & $10-20 \mathrm{kPa}$ & $20-40 \mathrm{kPa}$ \\
\hline $\max \left(e_{\text {spec }}\right)[\mathrm{kPa}]$ & 0.9 & 2.2 & 4.4 & 9.9 \\
$\max \left(e_{\text {univ }}\right)[\mathrm{kPa}]$ & 1.0 & 2.4 & 4.8 & 10.9 \\
\hline
\end{tabular}

TABLE II: Maximum errors between sensor value and reference pressure for a typical taxel of prototype $C$

errors for the typical taxel of prototype $C$ are shown. Using the universal model with just one fit function still provides low sensor errors. However, sensor errors can be higher for single taxels. The errors for the worst taxel are shown in Tab. III

\section{Hysteresis and Drift}

We applied multiple load cycles on one taxel of prototype $C$ to analyze the hysteresis effects of the piezoresistive material. Each load cycle has a different maximum pressure. The result is shown in Fig. 7 . At low pressures, the hysteresis effect is quite low. However, it may affect the measurement by up to $15 \mathrm{kPa}$ for higher pressures. Although our current sensor model does not include the hysteresis, this is in general suitable to further improve the accuracy of pressure sensing.

\begin{tabular}{lllll}
\hline Range: & $0-5 \mathrm{kPa}$ & $5-10 \mathrm{kPa}$ & $10-20 \mathrm{kPa}$ & $20-40 \mathrm{kPa}$ \\
\hline $\max \left(e_{\text {spec }}\right)[\mathrm{kPa}]$ & 1.4 & 3.7 & 7.9 & 14.4 \\
$\max \left(e_{\text {univ }}\right)[\mathrm{kPa}]$ & 1.6 & 4.7 & 11.8 & 24.8 \\
\hline
\end{tabular}

TABLE III: Maximum errors between sensor value and reference pressure for the worst taxel of prototype $C$

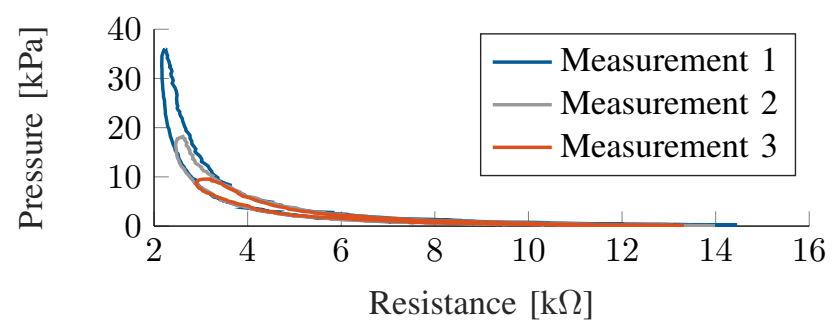

Fig. 7: Hysteresis of prototype $C$
In order to analyze the drift over time, prototype $C$ is loaded with a weight to ensure constant loads. The median drift of the sensed pressure over all taxels is $0.26 \mathrm{kPa} / \mathrm{s}$. This reduces the absolute accuracy for long-time static loads. However, the drift effect is equal for all taxels (standard deviation of $0.08 \mathrm{kPa} / \mathrm{s}$ ), i.e. spatial localization of contacts is still accurate.

\section{E. Spatial Resolution}

As we currently do not interpolate between the sensor data of the taxels, the spatial resolution is generally a function of the number of taxels per area. We were able to successfully produce prototypes with a taxel distance of $5 \mathrm{~mm}$. A video of the sensor output of prototype $C$ for different loads and contact scenarios is available at https://youtu.be/ HLdVkaF 9ZR4/

\section{Discussion}

Several prototypes were analyzed in the foregoing section. Prototype $C$, which is made out of a cotton fabric and machine-sewed steel threads showed the lowest threshold pressure and highest accuracy. Prototypes $A$ and $B$ can be made by hand, but the manufacturing effort is high for large areas and accuracies are lower. As prototype $C$ only requires a sewing machine and can be easily manufactured, it is selected as the final prototype. The sensor patch has a maximum activation threshold (over all taxels) of only $0.7 \mathrm{kPa}$, which is significantly below the values found in literature. The thresholds for comparable flexible sensors are $10-20 \mathrm{kPa}$ in [19], $15 \mathrm{kPa}$ in [18], $15.8-158 \mathrm{kPa}$ in [17] as well as $\approx 3-4 \mathrm{kPa}$ in [13].

We did not analyze the relationship between the sensor accuracy and the number of taxels, however we expect similar results for a larger number of taxels. Furthermore, the influence of parameters such as the thickness of the polymeric foil, the thread tension and thickness were not considered. Therefore, even better designs may be reached with further experiments in this direction.

The calibration model with specific parameter sets leads to very low errors in the sensed pressure as it considers local differences between the taxels. A universal calibration model, which was generated from all specific models, leads to almost the same accuracy for a typical taxel on the sensor patch. Although not investigated in the paper at hand, we expect this model to be applicable to other sensor units based on this design. In this case, a further calibration of the sensor patches is not necessary. Alternatively, the universal model can be adapted to each sensor unit by selected measurements.

The detected hysteresis leads to errors for higher pressures. This might be improved by an extended inverse hysteresis model. Furthermore, the sensor values drift over time. As the drift is equal for all taxels, we expect no negative impact on the accuracy of interpolation algorithms. With the current readout circuit only low update rates can be achieved. We expect the sensor to work with different and scalable readout electronics. 
With the described properties, this sensor is applicable to general tactile sensing in robotics. Due to the very low force thresholds, it may be used for collision detection or even on fingertips of a robotic hand. Despite the low-cost design, high accuracy of the sensed pressure is reached with a simple model. The design allows customization of spatial resolution, shape and position of the taxels.

\section{CONCLUSION}

In our contribution, we presented a flexible tactile sensor design, which is made of widely available and low-cost materials and can be manufactured with low effort. Due to the high flexibility, a bending radius of $5 \mathrm{~mm}$ is feasible. The material costs are $60-100 € / \mathrm{m}^{2}$, depending on the spatial resolution. As the sensor patch consists of fabrics with stainless steel threads, the spatial resolution can be customized and the sensor may be cut to arbitrary shapes. Experimental evaluation of the design shows activation thresholds of just $0.7 \mathrm{kPa}$. Furthermore, pressure sensing on the taxels is accurate in the range of $0-40 \mathrm{kPa}$. Pressures of up to $70 \mathrm{kPa}$ can be detected reliably. For the future, we plan to investigate on the use of an universal calibration model for different units of these sensor patches. In addition, we are going to increase spatial resolution via interpolation. Furthermore, we will focus on the scalability and readout-electronics of this tactile skin. We hope that other researchers in the field of robotics will rebuild this easy design in order to equip more robots with the sense of touch.

\section{APPENDIX}

\begin{tabular}{ll}
\hline Prototype Description & Drawbacks \\
\hline $\begin{array}{l}\text { Similar to prototype } A \text {, but with } \\
\text { a silicone cast instead of the neo- } \\
\text { prene cover }\end{array}$ & High pressure thresholds \\
\hline
\end{tabular}

Similar to prototype $C$, but with Difficult to manufacture the threads directly attached to the polymer using an adhesive foil and neoprene cover

Similar to prototype $B$, but lami- Air between the contacts nated with insulating foil instead of High bending radius the neoprene cover

TABLE IV: A list of earlier design prototypes and their drawbacks

\section{REFERENCES}

[1] D. Um, B. Stankovic, K. Giles, T. Hammond, and V. Lumelsky, "A modularized sensitive skin for motion planning in uncertain environments," in IEEE International Conference on Robotics and Automation, vol. 1, 1998.

[2] G. Cannata, M. Maggiali, G. Metta, and G. Sandini, "An embedded artificial skin for humanoid robots," in IEEE International Conference on Multisensor Fusion and Integration for Intelligent Systems, 2008.

[3] C. Schürmann, R. Koiva, R. Haschke, and H. Ritter, "A modular highspeed tactile sensor for human manipulation research," in IEEE World Haptics Conference, 2011.

[4] W. Dan Stiehl and C. Breazeal, "A sensitive skin for robotic companions featuring temperature, force, and electric field sensors," in IEEE/RSJ International Conference on Intelligent Robots and Systems, 2006.
[5] P. Mittendorfer, E. Dean, and G. Cheng, "3D Spatial Self-organization of a Modular Artificial Skin," in IEEE/RSJ International Conference on Intelligent Robots and Systems, 2014.

[6] K. Weiss and H. Wörn, "Resistive Tactile Sensor Matrices using InterElectrode Sampling," in IEEE Industrial Electronics Conference, 2005.

[7] F. Bergner, P. Mittendorfer, E. Dean-Leon, and G. Cheng, "Eventbased signaling for reducing required data rates and processing power in a large-scale artificial robotic skin," in IEEE/RSJ International Conference on Intelligent Robots and Systems, 2015.

[8] R. Koiva, M. Zenker, C. Schürmann, R. Haschke, and H. J. Ritter, "A highly sensitive 3D-shaped tactile sensor," in IEEE/ASME International Conference on Advanced Intelligent Mechatronics, 2013.

[9] G. Büscher, M. Meier, G. Walck, R. Haschke, and H. J. Ritter, "Augmenting Curved Robot Surfaces with Soft Tactile Skin," in IEEE/RSJ International Conference on Intelligent Robots and Systems, 2015.

[10] V. A. Ho, S. Hirai, and K. Naraki, "Fabric Interface with Proximity and Tactile Sensation for Human-Robot Interaction," in IEEE/RSJ International Conference on Intelligent Robots and Systems, 2016.

[11] T. Hoshi and H. Shinoda, "A Tactile Sensing Element for a Whole Body Robot Skin," Symposium on Robotics, pp. 1-6, 2005.

[12] T. Bhattacharjee, A. Jain, S. Vaish, M. D. Killpack, and C. C. Kemp, "Tactile Sensing over Articulated Joints with Stretchable Sensors," in IEEE World Haptics Conference, 2013.

[13] G. H. Büscher, R. Kõiva, C. Schürmann, R. Haschke, and H. J. Ritter, "Flexible and stretchable fabric-based tactile sensor," Robotics and Autonomous Systems, vol. 63, no. P3, pp. 244-252, 2015.

[14] K. Kim, K. R. Lee, D. S. Lee, N.-K. Cho, W. H. Kim, K.-B. Park, H.-D. Park, Y. K. Kim, Y.-K. Park, and J.-H. Kim, "A silicon-based flexible tactile sensor for ubiquitous robot companion applications," Journal of Physics: Conference Series, vol. 34, no. 1, p. 399, 2006.

[15] C. Cho and Y. Ryuh, "Fabrication of flexible tactile force sensor using conductive ink and silicon elastomer," Sensors and Actuators A: Physical, vol. 237, pp. 72-80, 2016.

[16] H.-K. Lee, S.-I. Chang, and E. Yoon, "A Flexible Polymer Tactile Sensor: Fabrication and Modular Expandability for Large Area Deployment," Journal of Microelectromechanical Systems, vol. 15, no. 6, pp. 1681-1686, 2006.

[17] V. Duchaine, N. Lauzier, M. Baril, M.-A. Lacasse, and C. Gosselin, "A Flexible Robot Skin for Safe Physical Human Robot Interaction," in IEEE International Conference on Robotics and Automation, 2009.

[18] Y.-L. Park, B. Chen, and R. J. Wood, "Design and Fabrication of Soft Aftificial Skin using Embedded Microchannels and Liquid Conductors," IEEE Sensors Journal, vol. 12, no. 8, pp. 2711-2718, 2012.

[19] M. W. Strohmayr, H. Wörn, and G. Hirzinger, "The DLR Artificial Skin Step I: Uniting Sensitivity and Collision Tolerance," in IEEE International Conference on Robotics and Automation, 2013.

[20] J. Ulmen and M. Cutkosky, "A robust, Low-Cost and Low-Noise Artificial Skin for Human-Friendly Robots," in IEEE International Conference on Robotics and Automation, 2010.

[21] A. Kheddar and A. Billard, "A Tactile Matrix for Whole-Body Humanoid Haptic Sensing and Safe interaction," in IEEE International Conference on Robotics and Biomimetics, 2011.

[22] M. Shimojo, A. Namiki, M. Ishikawa, R. Makino, and K. Mabuchi, "A Tactile Sensor Sheet Using Pressure Conductive Rubber With Electrical-Wires Stitched Method," IEEE Sensors Journal, vol. 4, no. 5, pp. 589-596, 2004.

[23] M. A. Lacasse, V. Duchaine, and C. Gosselin, "Characterization of the Electrical Resistance of Carbon-Black-Filled Silicone: Application to a Flexible and Stretchable Robot Skin," in IEEE International Conference on Robotics and Automation, 2010.

[24] S. Seo, S. Kim, J. Jung, R. Ma, S. Baik, and H. Moon, "Flexible Touch Sensors Made of Two Layers of Printed Conductive Flexible Adhesives," Sensors (Switzerland), vol. 16, no. 9, pp. 1-13, 2016.

[25] Y. Ohmura, Y. Kuniyoshi, and A. Nagakubo, "Conformable and Scalable Tactile Sensor Skin for Curved Surfaces," in IEEE International Conference on Automation and Robotics, 2006.

[26] M. Strohmayr, H. Saal, A. Potdar, and P. van der Smagt, "The DLR Touch Sensor I : A Flexible Tactile Sensor for Robotic Hands based on a Crossed-Wire Approach," in IEEE/RSJ International Conference on Intelligent Robots and Systems, 2010. 